\title{
Preparation of Keratin and PEO Blended Nanofibers Applied to Air Filtration
}

\author{
Cheng HUANG ${ }^{a}$, Xu-Hong YANG ${ }^{\text {b.* }}$ \\ National Engeering Laboratory for Modern silk, College of Textile and Clothing Engineering, \\ Soochow University \\ ahuangchenghc@163.com, byangxuhong@suda.edu.cn \\ *Xu-Hong YANG
}

Keywords: Keratin, PEO, Electrospinning, Air filtration

\begin{abstract}
The keratin was extracted from the wool by sodium sulfite dissolving system. And then different ratios of keratin/polyethylene oxide (PEO) formic acid solution were prepared. The rheological properties and conductivity of solution were studied. The influence of the proportion of keratin/PEO formic acid solution on the nanofibers' morphology was investigated. Finally, the nanofiber membences were composited on polypropylene nonwovens, the influence of spinning time on the air filtration was discussed.
\end{abstract}

\section{Introduction}

The wool resource wastes seriously by the reason of disposing the poor quality raw wools not fitting for spinning, processing loss in textile industry and so on. These wastes contain a large number of keratin. If the discarded keratin can be recycled, we can not only alleviate the current shortage of wool resources, but also reduce the pollution of the environment. Regenerated keratin has useful properties such as biocompatibility and biodegradability. Moreover, keratin materials can absorb heavy metal ions, formaldehyde and other volatile organic compounds. Keratins are proteins characterised by high stability and low solubility due to -S-S- cross-linking between cysteine amino acid residues[1]. The main purpose of physical blending of polymers is to improve the properties and processability of existing polymers[2]. Polyethylene oxide (PEO) is a kind of water soluble polymer compound which has no poison. In this work, keratin was blended with PEO in different proportion in order to improve its processability.

In recent years, the electrospinning process has gained increasing attention because it is a simple and effective method to produce nanofiber membranes[3].Keratin/PEO nanofibers were produced by electrospinning the blend formic acid solutions.

\section{Experiment}

Keratin was extracted from wool by sulphitolysis with sodium sulfide[4]. $5 \mathrm{~g}$ of wool fibers were cut into snippets of some millimeters and treated with $100 \mathrm{ml}$ of a solution containing urea(42g), Na2S $(1.9 \mathrm{~g})$ and sodium dodecyl sulfate $(0.8 \mathrm{~g})$ for $14 \mathrm{~h}$ at $35^{\circ} \mathrm{C}$. The mixture was dialysed against distilled water using a cellulose tube for 3 days at room temperature, changing the distilled water four times a day[5]. After the solution was filtered, the keratin powder was obtained at $-40^{\circ} \mathrm{C}$ in vacuum condition. Blend solutions of keratin/PEO 70/30, 60/40, 50/50, 40/60 and 30/70 were prepared by mixing the solutions of pure keratin and pure PEO with concentration of $8 \%(\mathrm{w} / \mathrm{w})$. The voltage was $20 \mathrm{kV}$, the receiving distance was $14 \mathrm{~cm}$, the flow rate was $0.2 \mathrm{ml} / \mathrm{h}$. Polypropylene nonwovens $\left(20 \mathrm{~g} / \mathrm{m}^{2}\right)$ was used to compose with nanofiber membranes to improve the mechanical properties. Membranes were obtained by electronspinning the blend solutions of keratin/PEO $50 / 50$ at $20 \mathrm{kV}, 14 \mathrm{~cm}$ and $0.2 \mathrm{ml} / \mathrm{h}$.

Viscosity of the blend solutions was measured by an AR2000 rheometer (40mm diameter and 2 angle ). Conductivity of mixed solution was measured with conductivity meter (DOS-11A).

The morphologies of the wool keratin particles and the fibrous membrane were investigated by a scanning electron microscopy (S4800 Japan). The specimens were coated with gold using a sputter 
coater and their morphology was observed under the SEM at an acceleration voltage of $5 \mathrm{kV}[6]$.

Air filtration performance of composite membrane was tested by 8130 type automatic filter tester, and we used sodium chloride aerosol whose median grain size was $75 \mathrm{~nm}$ as a particulate filter and test air flow rate was $85 \mathrm{~L} / \mathrm{min}$.Then $\mathrm{S} 4800$ scanning electron microscope (SEM) was used to observe changes of the morphology.

\section{Results and Discussion}

\subsection{Rheology and Conductivity}

The rheological properties of the keratin/PEO formic acid solutions are shown in Fig.1a. Due to the loose molecular chain, the viscosity of keratin fomaid acid solution is very low. However, the relative molecular weight of PEO is 400000, there are more nodes between the molecules, so the viscosity of PEO fomaid acid solution is much higher. With the increase of shear rate, the shear viscosity decreases gradually.

As we can see in Fig.1b, pure keratin solution has higher conductivity because of polar amino acids[7]. With the increase of keratin, the viscosity of the solutions decreases gradually while the conductivity increases.

a

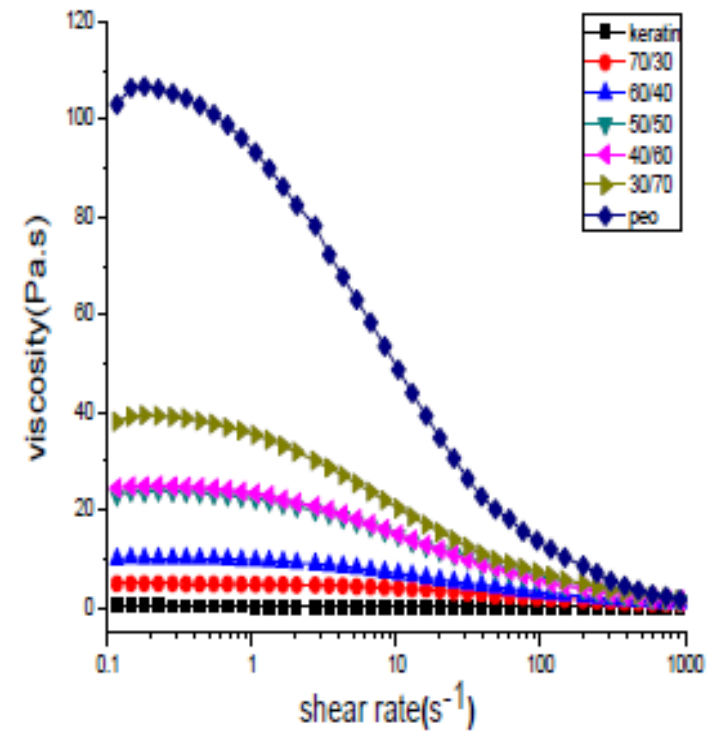

b

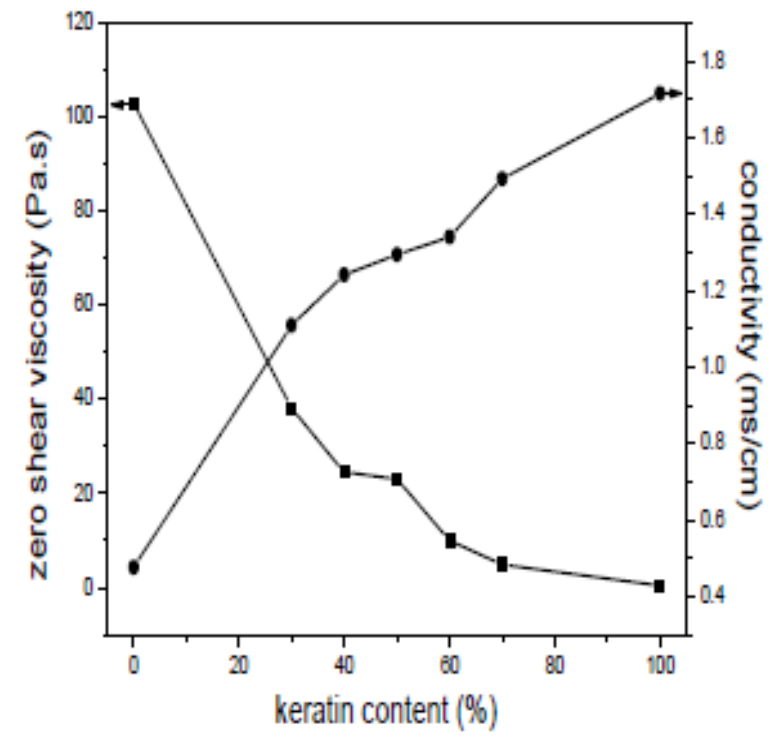

Fig.1 (a) Rheology of solutions, (b) Viscosity and conductivity of solutions

\subsection{Morphology}

SEM images of nanofiber prepared by different proportion solution are shown in Fig.2. The nanofibers are smooth and uniform, and there is no adhesion between nanofibers. The change of the proportion of keratin and PEO mainly resulted in changes of viscosity and conductivity of the mixed solutions. In the appropriate range, the decrease of viscosity and the increase of conductivity is conducive to the drawing of nanofibers. As summarized in Table 1, with the increase of keratin, the diameter of nanofibers decreases obviously. 


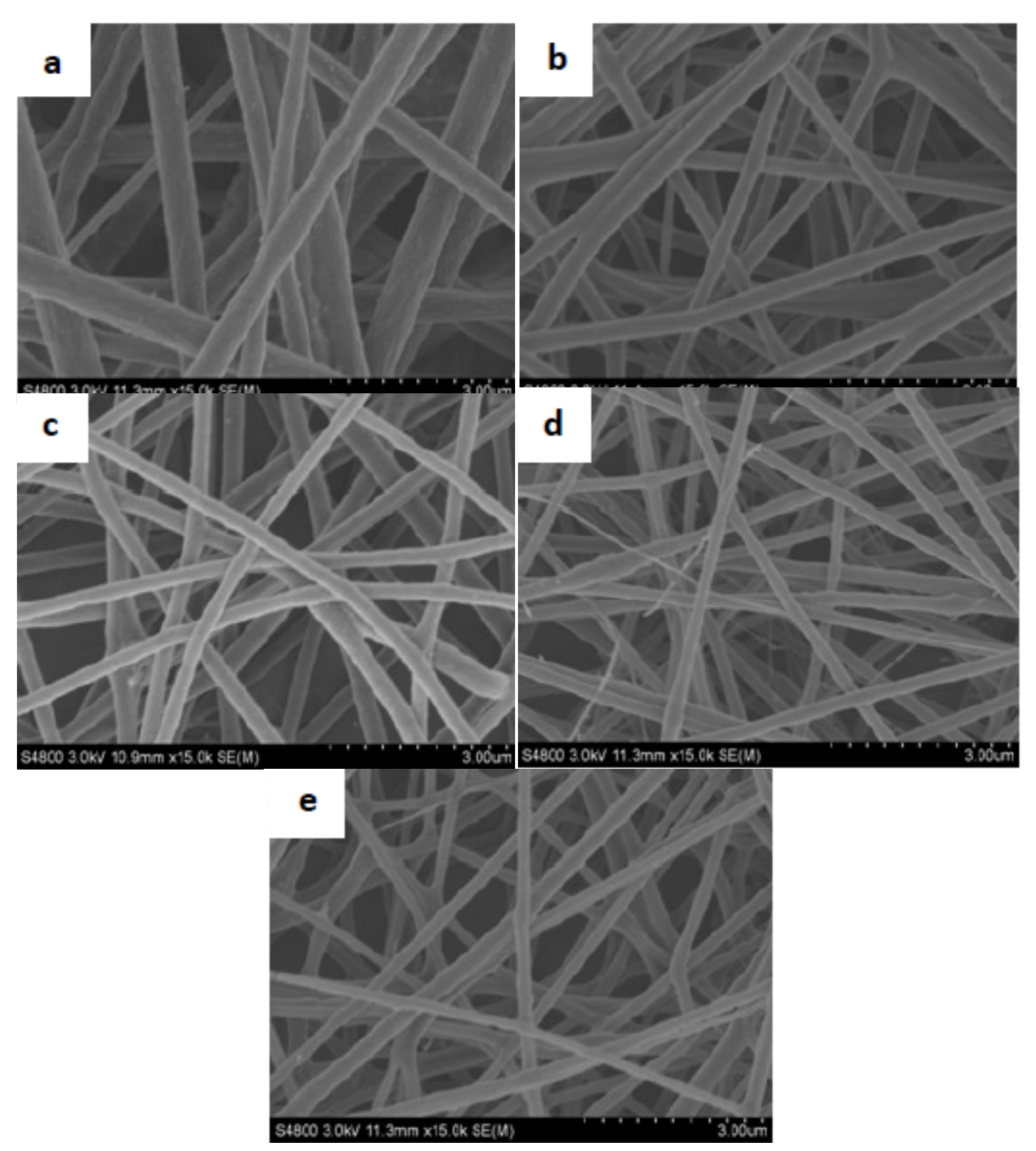

Fig.2 (a)Keratin/PEO 30/70 (b) Keratin/PEO 40/60 (c) Keratin/PEO 50/50

(d) Keratin/PEO 60/40 (e) Keratin/PEO 70/30

Table 1: Average diameter of different raito solutions

\begin{tabular}{|l|l|l|l|l|l|}
\hline Keratin/PEO & $30 / 70$ & $40 / 60$ & $50 / 50$ & $60 / 40$ & $70 / 30$ \\
\hline Average diameter $(\mathrm{nm})$ & 498.4 & 323.1 & 283.1 & 230.0 & 214.8 \\
\hline
\end{tabular}

\subsection{Air Filtration}

The air filtration of the samples with different spinning time are shown in Fig.3. As shown in Fig.3a, the penetration of polypropylene nonwoven fabric is near 100 per cent. That means nonwoven fabric has very poor air filtration performance. When the spinning time was $1 \mathrm{~h}$, the transmission rate of the composite membrane decreased obviously. This is due to high specific surface area and strong adsorption capacity of nanofibers. With the increase of spinning time, the fiber layer became thicker, the gap between fibers became smaller. As a result, the penetration rate decreased, and the resistance increased gradually, air filtration performance became batter. The recommend final pressure drop of fine filter is $450 \mathrm{~Pa}$. When the spinning time was $4 \mathrm{~h}$, the resistance was $352.7 \mathrm{~Pa}$, and the penetration was about $93.9 \%$, so the air filtration performance was quite well. 


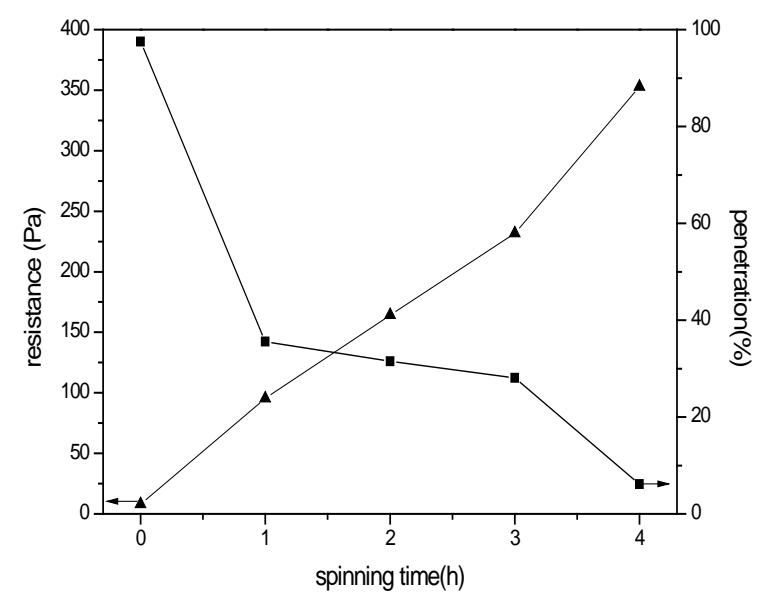

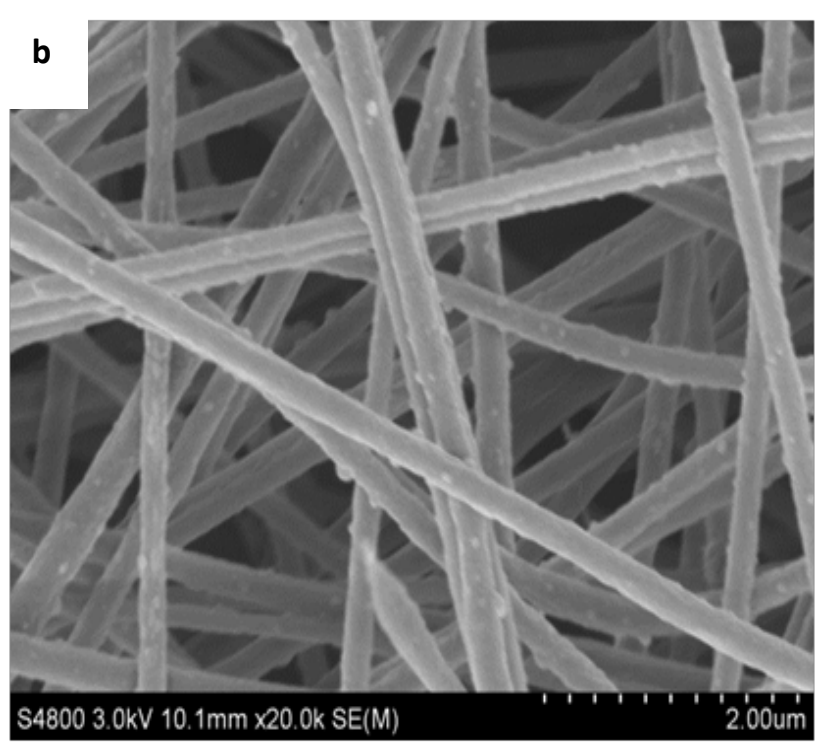

Fig.3 (a) Penetration and resistance, (b) SEM image of composite membrane

Fig.3b shows morphology of the composite membrane after filtration. Filtering mechanism is mainly divided into diffusion effect, interception effect, momentum effect, sieve effect, electrostatic effect etc. It can be seen that diameter of aerosol is much smaller than that of the nanofibers, so the sieve effect is very little, while diffusion effect, interception effect and momentum effect play an important role in filtration.

\section{Summary}

In this work, wool-derived keratin nanofiber membranes were prepared by the electrospinning keratin/PEO blended formic acid solutions. With the increase of keratin, the viscosity of the solutions and the diameter of nanofibers decreased gradually, while the conductivity increased. Compared to the single polypropylene nonwovens the air filtration performance of the composit materials of polypropylene nonwoven/nanofibers were obviously improved. And with the increasing spinning time the penetration rate of nano particulate decreased indicating that the air filtering effect became better. However, the filtration resistance increased gradually. When the spinning time was $4 \mathrm{~h}$, the composite membranes had the best air filtration performance.

The diameter is play an important role in air filtration, there are many other factors that affect the diameter of the nanofibers, such as mass fraction, voltage, rate of flow, spinning distance etc. Therefore, the future research may focus on the effect of spinning parameters on air filtration performance.

\section{References}

[1] Zoccola M, Aluigi A, Tonin C. Characterisation of keratin biomass from butchery and wool industry wastes[J]. Journal of Molecular Structure, 2009, 938(1):35-40.

[2] Kausar A, Zulfiqar S, Sarwar M I. Nanoblends of PMMA/aramid: A study on morphological and physical properties[J]. Solid State Sciences, 2013, 24(10):36-43.

[3] Aluigi A, Corbellini A, Rombaldoni F, et al. Morphological and structural investigation of wool-derived keratin nanofibres crosslinked by thermal treatment[J]. International Journal of Biological Macromolecules, 2013, 57(6):30-37.

[4] Candela MP, Martinez JM, Macia RT. Chromium(VI) removal with activated carbons[J]. Water Research, 1995, 29(9):2174-2180.

[5] Aluigi A, Tonetti C, Vineis C, et al. Adsorption of copper (II) ions by keratin/PA6 blend 
nanofibres[J]. European Polymer Journal, 2011, 47(9): 1756-1764.

[6] Li J, Li Y, Li L, et al. Preparation and biodegradation of electrospun PLLA/keratin nonwoven fibrous membrane[J]. Polymer Degradation \& Stability, 2009, 94(10):1800-1807.

[7] Aluigi A, Vineis C, Varesano A, et al. Structure and properties of keratin/PEO blend nanofibres[J]. European Polymer Journal, 2008, 44(8): 2465-2475. 